\title{
Structure and Permeability of Porous Silicon Investigated by Self-Diffusion NMR Measurements of Ethanol and Heptane
}

\author{
J. Puibasset ${ }^{1 *}$, P. Porion ${ }^{1}$, A. Grosman ${ }^{2}$ and E. Rolley ${ }^{3}$ \\ ${ }^{1}$ Interfaces, Confinement, Matériaux et Nanostructures, CNRS UMR 7374, Université d'Orléans, 16 rue de la Férollerie, \\ 45071 Orléans Cedex 02 - France \\ 2 Institut des NanoSciences de Paris, CNRS, Sorbonne Universités, UPMC Univ. Paris 6, 4 place Jussieu, 75005 Paris - France \\ ${ }^{3}$ Laboratoire de Physique Statistique de I'ENS, CNRS, Univ. Paris 6 \& 7, 24 rue Lhomond, 75005 Paris - France \\ e-mail: puibasset@cnrs-orleans.fr - porion@cnrs-orleans.fr - annie.grosman@insp.upmc.fr - rolley@lps.ens.fr \\ * Corresponding author
}

\begin{abstract}
The adsorption and phase transitions of confined fluids in nanoporous materials have been studied intensely because of both their fundamental interest and their crucial role in many technologies. Questions relating to the influence of the confinement of fluids, and the disorder or elastic deformation of porous solids on the liquid-gas phase transition are still under debate. Model systems are needed to understand the adsorption phenomenon. In this context, Porous Silicon (PoSi), which is a single crystal obtained by etching a (100) silicon wafer is an excellent candidate. Indeed, it consists of non-connected tubular pores running parallel to the [100] axis perpendicular to the wafer surface, with transverse sections with a polygonal shape of nanometric size whose areas are widely distributed. Once detached from the wafer, free PoSi membranes can be considered a nanoscale disordered honeycomb. Adsorption/desorption experiments have been performed to characterize the structure: they have shown that evaporation occurs collectively, an intriguing observation generally associated with a disordered pore structure with many interconnections through narrow necks. The characterization of fluid mobility inside the pores should give complementary information about the pore structure and topology. This paper focuses on the dynamics of a fluid confined inside the structure of porous silicon, and in particular the self-diffusion measurements (pulsed field gradient spin echo Nuclear Magnetic Resonance (NMR)). The results show a strong anisotropy of the selfdiffusion tensor, as expected in this highly anisotropic structure. However, a non-zero self-diffusion in the directions perpendicular to the pore axis is observed. In order to interpret these puzzling results, molecular and Brownian dynamics calculations are underway.
\end{abstract}

Résumé - Structure et perméabilité du silicium poreux : étude de l'autodiffusion de l'éthanol et de l'heptane par RMN - L'adsorption et les transitions de phase de fluides confinés dans des nanopores sont étudiées pour leur intérêt fondamental et technologique. En particulier, l'influence du confinement du fluide, du désordre ou des déformations élastiques du milieu poreux sur la transition liquide-vapeur sont toujours débattus. Des systèmes modèles sont requis pour mieux comprendre ces phénomènes en lien avec l'adsorption. Dans ce contexte, le silicium poreux obtenu par attaque chimique de la surface (100) d'un wafer de silicium parait être un excellent candidat. Il est en effet constitué de pores tubulaires non-connectés et parallèles à l'axe [100] perpendiculaire à la surface du wafer ; ces pores sont à section polygonale de taille nanométrique, avec une distribution en taille 
large. La membrane de silicium poreux, une fois détachée du wafer peut être vue comme une structure en nid d'abeilles désordonné. Des mesures d'isothermes d'adsorption/désorption ont été réalisées pour caractériser la structure : elles ont montré que l'évaporation a lieu de manière collective, une observation inattendue, généralement interprétée comme étant la signature d'un milieu poreux désordonné avec de nombreuses interconnexions étroites. La caractérisation de la mobilité du fluide à l'intérieur des pores devrait fournir des informations complémentaires sur la structure et la topologie des pores. Ce papier s'intéresse à la dynamique d'un fluide confiné dans le silicium poreux, en particulier par mesure du coefficient d'autodiffusion par Résonance Magnétique Nucléaire (RMN) (gradients de champs pulsés). Les résultats montrent une forte anisotropie du tenseur de diffusion, en accord avec la structure très anisotrope du milieu. Toutefois, une diffusion non-nulle est observée dans les directions perpendiculaires à l'axe des pores. L'interprétation de ces résultats se fera à l'aide de calculs de dynamique Brownienne et moléculaire.

\section{INTRODUCTION}

Information about the morphology, structure and topology of the void space of a porous material is important in understanding and predicting the dynamics of confined fluids. Applications and situations where such information is of interest are, among others, catalysis, selective adsorption, fuel cells, gas shale, aquifers, etc. Dynamics of confined fluids includes self-diffusion and convection. The former is associated with random motion, and takes place without any driving force. The latter is associated with a coherent motion of molecules due to a chemical potential gradient (driving force). Both are influenced by the structure and topology of the porous material, as well as the interactions between the fluid molecules and the solid surface. It is important to note that self-diffusion and convection are quite different processes which are not sensitive to the same morphological and topological characteristics of the porous medium $[1,2]$.

Porous materials develop a large surface to volume ratio, in particular when their pores are nanometric. A strong effect on diffusion and transport are thus expected, since the molecules spend a long time in the vicinity of a surface [3, 4]. A full characterization of nanoporous materials is thus required to understand the dynamics of the confined fluid. At the same time, it is a very difficult task, since very informative techniques like 3D tomography have a resolution of a few micrometers at best. Microscopy scales down to a few nanometers, but in most cases the topology remains hardly accessible since the observations are two-dimensional [5]. The characterization of nanoporous materials is thus generally performed by analysing the adsorption-desorption isotherms of simple gases [6]. These measurements, based on the pore-size-dependent equilibrium properties of the confined fluid, allow us to extract the pore size distribution by means of an appropriate model. However, little can be inferred about the pore morphology and topology. Permeation and diffusion measurements thus appear to offer a complementary source of information in the characterization of porous materials.

Porous Silicon (PoSi) nanostructures [7] have been extensively studied for their optical properties and potential compatibility with silicon based electronics. Their large surface-to-volume ratio is also an advantage for the development of gas-sensing devices or for applications involving fuel cells $[8,9]$. A large variety of pore morphologies and a large range of porosities can be obtained depending on the doping and etching conditions [10]. The PoSi samples used in this work have been obtained by electrochemical etching of monocrystalline (100)-oriented, highly p-doped Si wafers. In these conditions, the etching leads to straight pores aligned with the [001] axis [11]. PoSi thus appears to be a model porous material to study various fundamental issues, including mechanics, adsorption or transport properties [11-16].

The adsorption-desorption isotherms of simple fluids in PoSi exhibit hysteresis [12], as expected for nanometric pores. However, desorption is sharper than expected, suggesting that pore-emptying occurs in a collective process. This is usually attributed to the connectivity of the pore network [17], but pores in PoSi are disconnected [12]. Thus, the apparent collective behavior has been attributed to disorder inside each pore [18] or to the adsorption-induced mechanical deformation of the pore walls [19].

The goal of the present work is to propose a preliminary study of the permeation and diffusion properties of ethanol and $n$-heptane confined in PoSi. The high aspect ratio of the pores induces a strong anisotropy of the material, with expected original consequences for the dynamics of the fluid. Furthermore, the dynamics is sensitive to the pore topology, and could provide information useful in proposing complementary interpretations of the puzzling adsorptiondesorption isotherms. 


\section{SAMPLE PREPARATION AND EXPERIMENTAL SETUPS}

The PoSi samples used in this work have been obtained by electrochemical etching of monocrystalline (100)-oriented, highly boron $(0.003 \Omega . \mathrm{cm})$ doped Si wafers at a current density of $20 \mathrm{~mA} / \mathrm{cm}^{2}$. The etching solution was a $3: 1$ mixture of hydrofluoric acid and ethanol solutions. These conditions lead to a porosity of $51 \%$ [10]. The PoSi thickness is controlled by the etching time, and ranges typically between 10 and $100 \mu \mathrm{m}$. The morphology of these layers (Fig. 1) can be described as a honeycomb structure, with straight pores perpendicular to the Si wafer surface separated from each other by Si single crystal walls of uniform thickness $(5-6 \mathrm{~nm})$. The pore cross-section is roughly polygonal in shape and the pore radius distribution is large, $6.5 \pm 3 \mathrm{~nm}$.

An important issue regarding the interpretation of adsorption isotherms is to know whether the pores are interconnected. To check this point, an oxidation experiment has been performed with a specific oxygen isotope, allowing its localization in the sample by nuclear reaction. A supported sample has been used, which was partially covered with aluminium. In that situation, the oxidation (performed at $300^{\circ} \mathrm{C}, 12 \mathrm{mbar}$ and for $1 \mathrm{~h}$ ) may occur in the pores under the cap only if there are interconnections between the pores. The experiment shows that there is no measurable oxidation under the cap. The interconnections are thus negligible. More details can be found in Reference [12].

The overall size of the samples used in this study is 100 micrometers along the pore axis and a few centimeters transverse to the pores: the samples detached from the wafer do actually look like rigid thin membranes. The samples are used as prepared for permeation experiments, while for diffusion measurements they are first saturated with ethanol or $n$-heptane and kept in a sealed tube.

\subsection{Diffusion Measurements}

Diffusion is a consequence of a rapid de-correlation in the motion of molecules due to interactions with other molecules. The mean square displacement tends to be proportional to time, thus defining the diffusion coefficient. In a porous material, the molecules are restricted to evolving in the accessible volume: the mean square displacement remains proportional to time, but with an effective diffusion coefficient which is smaller than in bulk. This is because the molecules have to travel a larger distance through tortuous channels $[1,2,20]$.

In our case, the porous material is also anisotropic, and a diffusion tensor $D_{\alpha \beta}$ can be defined according to:

$$
\lim _{t \rightarrow \infty}\left\langle r_{\alpha} r_{\beta}\right\rangle=2 D_{\alpha \beta} t
$$

where $\alpha$ and $\beta$ run over the three space coordinates, and $r$ is the position of the particle at time $t$. This tensor is symmetric,
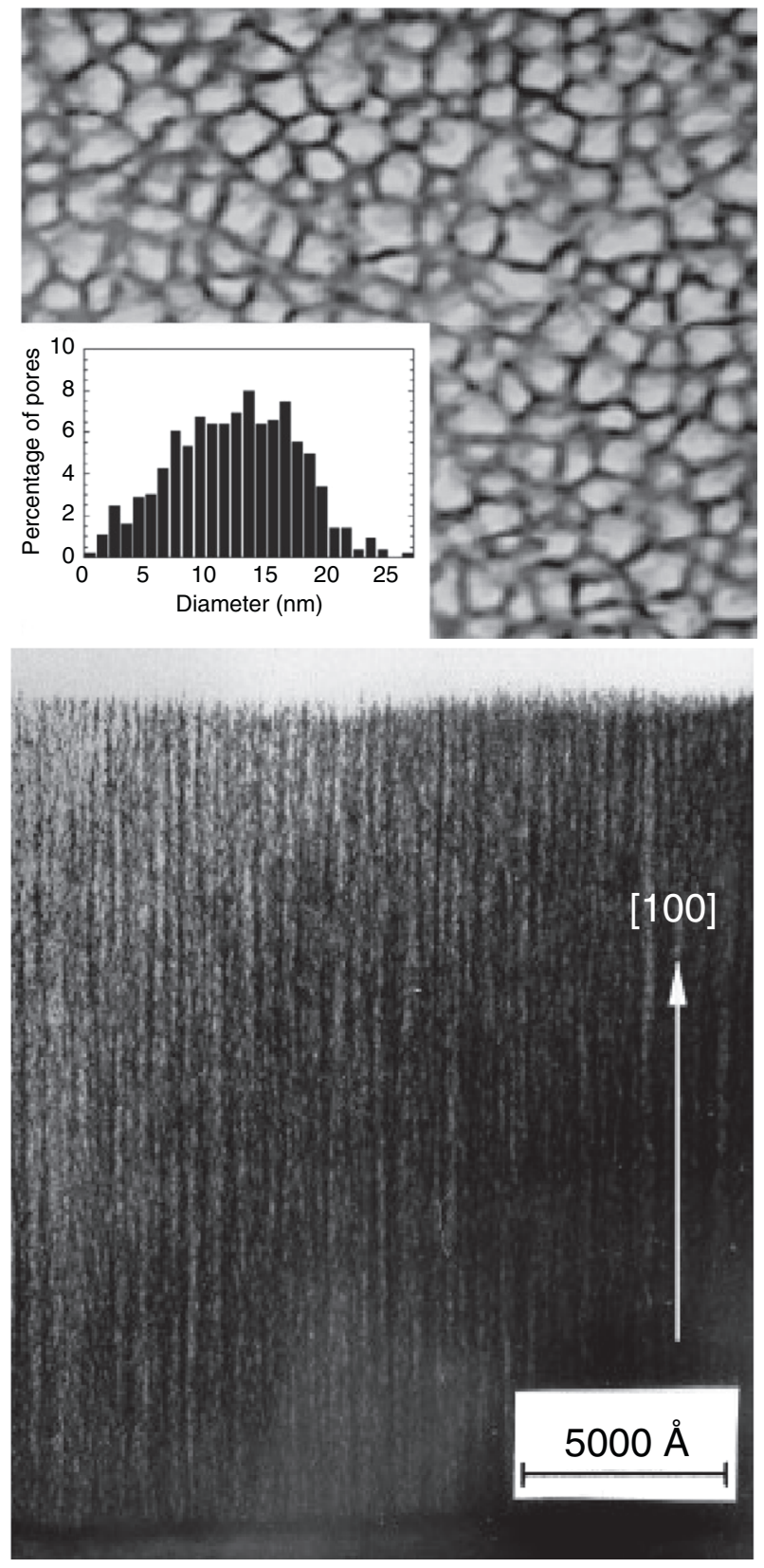

Figure 1

Transmission electron microscopy views of porous silicon samples with $50 \%$ porosity. Top: plane view; bottom: side view.

and can be diagonalized. This provides the main axis of the material. Important information about the porous structure can be inferred from the measurement of this quantity.

The Pulsed Field Gradient Spin Echo Nuclear Magnetic Resonance (PFGSE NMR) is able to perform directly this measurement for fluids confined in porous materials $[21,22]$. More specifically, the technique measures the diffusion coefficient of nuclear spins, in particular that of 


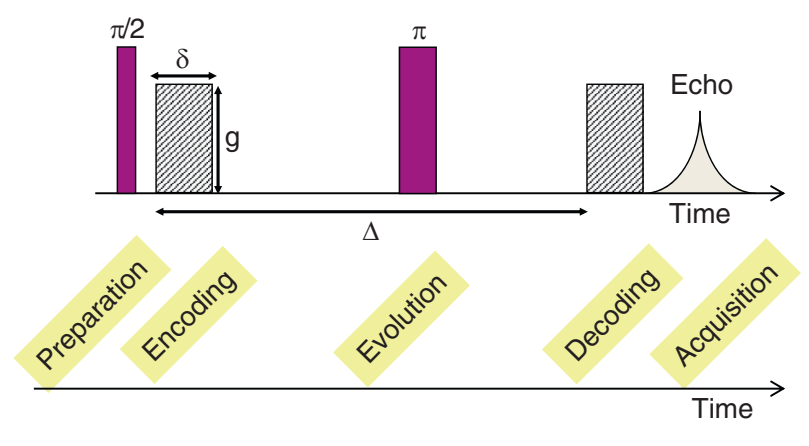

Figure 2

Schematic view of the stimulated echo pulse sequence used for the measurement of the self-diffusion tensors.

hydrogen. ${ }^{1} \mathrm{H}$ pulsed field spin-echo attenuation was used to measure the mobility of ethanol and heptane molecules within PoSi. The diffusion-weighed stimulated echo pulse sequence has been used (Fig. 2) with an echo attenuation according to [22]:

$$
\frac{I(q)}{I(0)}=\exp \left[-4 \pi^{2} \sum_{\alpha, \beta} q_{\alpha} q_{\beta} D_{\alpha \beta}(\Delta-\delta / 3)\right]
$$

where $2 \pi \vec{q}=\gamma \delta \vec{g}, \vec{g}$ is the applied field gradient (varying between 0 and $1.5 \mathrm{Tm}^{-1}$ in magnitude), $\delta$ is its duration, $\Delta$ is the diffusion time, $\gamma$ is the gyromagnetic ratio of the nuclei, $D_{\alpha \beta}$ is the self-diffusion tensor, and $I(q)$ and $I(0)$ are the echo intensities measured with and without the field gradient, respectively. Since the same pulse sequence, using the same coherence pathway, is used for recording the echo intensities with and without the field gradient, they are both affected by the relaxation mechanisms of the molecule, leading to the same echo attenuation. As a consequence, the relaxation mechanisms do not contribute to the measured ratio $I(q) / I(0)$.

The diffusion experiments were performed on a Bruker DSX 100 spectrometer equipped with gradient coils in three perpendicular directions used to generate magnetic field gradients along any arbitrary direction. The maximum value of the magnetic field gradient $\left(1.5 \mathrm{Tm}^{-1}\right)$ corresponds to wavenumbers $q$ smaller than $2 \times 10^{5} \mathrm{~m}^{-1}$. The diffusion time has to be chosen so that the echo attenuation is important in order to reduce the uncertainty $(\Delta=50 \mathrm{~ms}$ in our experiments). The distance travelled by the molecules is thus of order $\mathrm{q}^{-1}$ i.e. $5 \mu \mathrm{m}$. This distance is much larger than the pore diameter, and much less than the pore length. The inplane measurement is thus sensitive to the diffusion at length scale larger than the pore diameter, while the measurement along the pore axis is performed within a time interval short

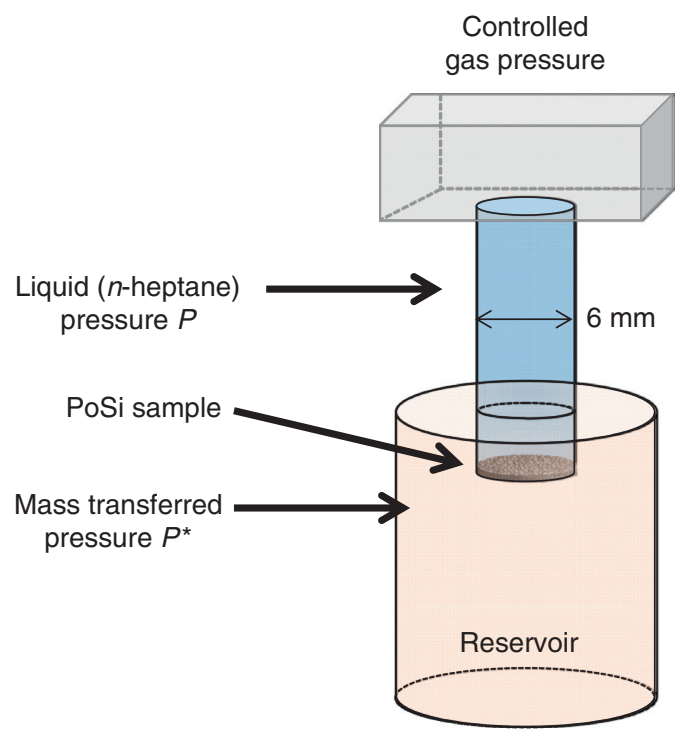

Figure 3

Schematic view of the setup used for the permeation measurements.

enough so that the molecule dynamics is not influenced by the pore ends. This unique technique has already been used to measure anisotropic diffusion tensor of confined fluids $[16,23-25]$.

\subsection{Permeation Measurements}

The permeation measurements have been performed using a home-made apparatus. The principle follows the standard technique: a liquid is pushed through the porous material, while the pressure difference across the material is controlled (Fig. 3). The sample is glued with epoxy (Stycast) at the mouth of an inox tube, whose inner diameter is $6 \mathrm{~mm}$. The system is at room temperature and vacuum pumped. Then the liquid is introduced and pressurized (controlled pressure $P$ ). The mass transferred is measured at various times by weighing. It has been checked that evaporation was negligible during the operation. All permeability measurements were performed with $n$-heptane.

\section{RESULTS AND DISCUSSION}

\subsection{Diffusion Measurements}

We performed our diffusion measurements in PoSi saturated with ethanol or heptane at $290 \mathrm{~K}$. Each sample, possessing dimensions of approximately $5 \mathrm{~mm}$ width and $15 \mathrm{~mm}$ 


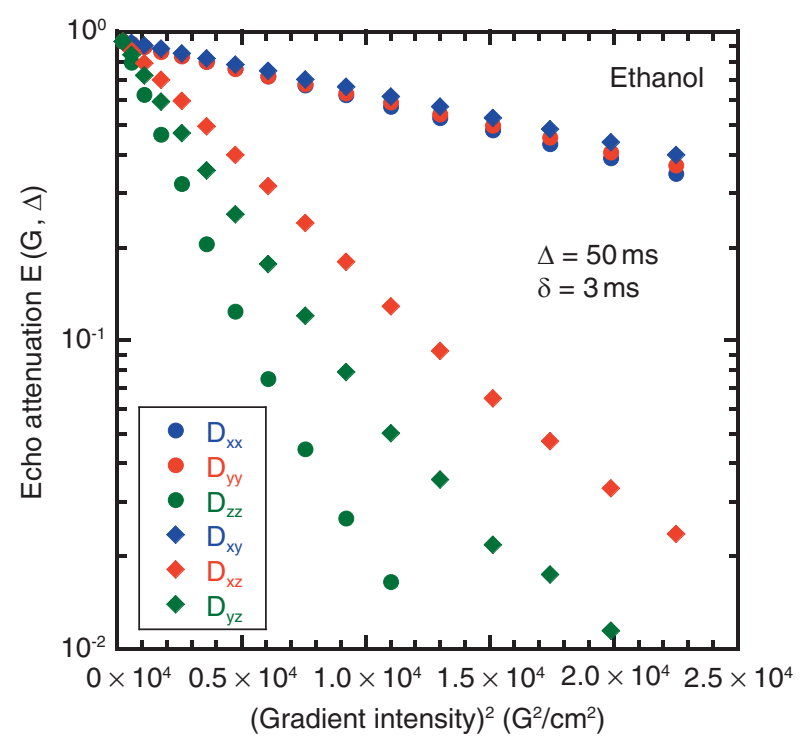

Figure 4

PFGSE NMR attenuations measured along the six independent axes for ethanol adsorbed in porous silicon.

length, is put into a $6 \mathrm{~mm}$ diameter tube suited for NMR measurements. The tubes are connected to a standard adsorption-desorption system. The samples were first outgassed, and then saturated by increasing the vapor pressure in the vessel. The procedure follows the usual adsorption isotherm measurement. It is stopped when the saturation is reached, and the tube containing the sample is sealed.

The PFGSE measurements are performed in six independent directions defining the diffusion tensor $D_{\alpha \beta}$ for both ethanol and heptane. These directions correspond to the three coordinate axes $x, y$ and $z$ (diagonal coefficients) and the three diagonals $x y, x z$ and $y z$ (off-diagonal terms). Note that the sample is positioned so that the pores are almost parallel to the $z$ axis. The corresponding attenuations are given in Figure 4 in semi-logarithmic scale. As can be seen, the curves are essentially linear, in agreement with the hypothesis of a diffusion process with Gaussian propagator. The six diffusion coefficients, deduced from the slopes, are significantly different: the system is thus anisotropic.

The diagonalisation of the tensor gives the three principal directors of the system and the associated diffusion coefficients (Tab. 1). For both fluids, a strong anisotropy is observed, corresponding to a uniaxial system, with the main axis parallel to the pore axis within experimental errors. As can be seen, the in-plane diffusion is dramatically attenuated compared to bulk diffusion (by two orders of magnitude), in agreement with the classical picture that the PoSi is made of
TABLE 1

Diffusion coefficients along the main axes after diagonalisation of the diffusion tensor $\left(10^{-10} \mathrm{~m}^{2} / \mathrm{s}\right)$

\begin{tabular}{c|c|c|c|c}
\hline & $D_{x}$ & $D_{y}$ & $D_{z}$ & $D_{\text {bulk }}$ \\
\hline$n$-heptane & 0.244 & 0.241 & 4.58 & 29.2 \\
\hline Ethanol & 0.113 & 0.104 & 1.35 & 9.8 \\
\hline
\end{tabular}

a collection of independent pores. However, a residual in-plane diffusion that emerges from the noise can be observed. The diffusion time $\Delta$ being $50 \mathrm{~ms}$, a molecule with a diffusion coefficient of $10^{-11} \mathrm{~m}^{2} / \mathrm{s}$ (as observed for ethanol) travels a distance of order 1 micrometer. This is much larger than the pore diameter of the largest pores. As a consequence, this diffusion has to be attributed to possible sporadic interconnections between pores or to a twodimensional diffusion on the external surface of the membrane. The former is disfavored by previous experiments, which show negligible interconnections between pores [12]. The second explanation relies on the fact that molecules close enough to the pore ends are in rapid exchange with the molecules freely diffusing on the external surface of the membrane. The whole population thus acquires an average diffusion coefficient parallel to the surface of the membrane, and thus perpendicular to the pore axis. More experiments are required to evaluate the validity of this hypothesis.

The diffusion along the pore axis is significantly reduced, for both liquids and with essentially the same factor of around 7. During the diffusion delay $\Delta=50 \mathrm{~ms}$ used in the experiment, a molecule with a diffusion coefficient of $4.6 \times 10^{-10} \mathrm{~m}^{2} / \mathrm{s}$ (as observed for $n$-heptane) travels a distance of order $7 \mu \mathrm{m}$. This distance is much less than the length of the pores. As a consequence, the observed attenuation of the diffusion constant is not due to the finite length of the pores. The purely geometric interpretation of this factor in terms of tortuosity leads to an effective increase in length by a factor of 2.6. This large value is not compatible with the straight tubes of PoSi. However, such high values have already been observed in linear pores, and several models have been proposed [15]. For instance, the presence of constrictions along the pores. We have performed Brownian Dynamics simulations which show that such constrictions are actually inefficient in reducing diffusion. It has also been proposed that the presence of strongly adsorbing sites or blind cavities could also reduce diffusion along the $z$ axis. Finally, one could also have pore obstruction with small interconnections. This represents a large spectrum of possibilities, some of them far from the simple picture of an assembly of linear pores. Complementary permeation measurements have thus been performed in order to help to discriminate between these models. 
TABLE 2

Samples, operating conditions and measurements for the permeation experiments

\begin{tabular}{c|c|c|c|c|c|c}
\hline Sample & $\begin{array}{c}\text { Age } \\
(\text { days })\end{array}$ & $\begin{array}{c}S \\
\left(\mathrm{~cm}^{2}\right)\end{array}$ & $\begin{array}{c}\Delta P \\
(\mathrm{bar})\end{array}$ & $\begin{array}{c}\Delta t \\
(\mathrm{~min})\end{array}$ & $\begin{array}{c}\Delta m \\
(\mathrm{~g})\end{array}$ & $\begin{array}{c}k \\
\left(\mathrm{~nm}^{2}\right)\end{array}$ \\
\hline $\mathrm{A}$ & $\sim 100$ & 0.283 & 1 & 451 & 0.067 & 0.063 \\
\hline $\mathrm{A}$ & $\sim 100$ & 0.283 & 1 & 1153 & 0.163 & 0.059 \\
\hline $\mathrm{B}$ & $\sim 7$ & 0.264 & 1.3 & 50 & 0.112 & 0.61 \\
\hline $\mathrm{C}$ & $\sim 1$ & 0.283 & 1.1 & 76 & 0.265 & 1.2 \\
\hline $\mathrm{C}$ & $\sim 7$ & 0.283 & 1.13 & 168 & 0.439 & 0.85 \\
\hline
\end{tabular}

\subsection{Permeation Measurements}

The permeation experiments have been performed for various samples and operating conditions: pressure, duration, etc. (Tab. 2). We have checked that the system follows Darcy's law, in particular the linearity of the flow rate with the pressure gradient in the system, and its independence from the duration of the experiment. The permeability can thus be defined as follows:

$$
k=\frac{Q \mu L}{S \phi \Delta P}
$$

where $Q$ is the volume flow rate, $\mu$ is the viscosity, $L$ and $S$ are the membrane thickness and surface respectively, $\phi=50 \%$ is the porosity, and $\Delta P$ is the pressure gradient across the membrane.

The effect of the sample age (time elapsed between the sample elaboration and the permeability measurement, where the sample is kept at room temperature and pressure) has been evaluated. Data show an important diminution in permeability with time (factor 2 after one week and 20 after 3 months). This evolution could be due to a spontaneous modification of the surface chemistry, and a possible oxidation after several weeks, which induces thickening of the walls. The largest value of permeability obtained for freshly prepared samples is $1.2 \mathrm{~nm}^{2}$.

Let us compare the permeability value with a simple model. Using the Hagen-Poiseuille equation, the expected permeability for a collection of identical tubes of radius $6.5 \mathrm{~nm}$ is $5.3 \mathrm{~nm}^{2}$, significantly larger than the measured $1.2 \mathrm{~nm}^{2}$. If one takes into account the pore size distribution, the obtained permeability is even larger since the largest pores contribute the most. Conversely, the experimental permeability we have measured may be translated into an effective hydraulic radius: this gives a radius equal to $3 \mathrm{~nm}$, i.e. among the smallest pore sizes. This suggests that the pores could either be strongly undulating or present significant constrictions or obstructions. Note that these interpretations seem hardly compatible with the etching process which most probably favors smooth walls parallel to crystallographic planes. Further investigations are necessary to improve our understanding of the results.

\section{CONCLUSION}

Diffusion and permeation measurements have been performed on PoSi membranes. PFGSE NMR shows that diffusion is strongly anisotropic, as expected for a honeycomb structure. However, strong unexpected attenuation is observed along the pore axis. This could be explained by surface roughness, or by the presence of blind cavities along the pores. Permeation measurements indicate a slow evolution of the PoSi membrane at room temperature, which may be due to a slow oxidation at ambient conditions. The largest permeability values, obtained for freshly prepared samples, are significantly lower than expected for a simple model made of parallel tubes. Regarding fluid flow in $10 \mathrm{~nm}$ large tubes, a surface roughness compatible with the etching process, which most probably favors smooth walls parallel to crystallographic planes, is not expected to have a large impact. More refined calculations are required to take into account the polygonal shape of the pores: this could severely influence the velocity profile in the pores and the corresponding expected permeation. Complementary microscopy observations could also provide useful information on the pore morphology.

\section{REFERENCES}

1 Bear J. (1972) Dynamics of fluids in Porous Media, Dover, New York.

2 Dullien F.A.L. (1979) Porous Media. Fluid Transport and Pore Structure, Academic, San Diego.

3 Levitz P., Korb J.P., Petit D. (2003) Slow dynamics of embedded fluid in mesoscopic confining systems as probed by NMR relaxometry, Eur. Phys. J. E 12, 1, 29-33.

4 Malek K., Coppens M.-O. (2001) Effects of surface roughness on self- and transport diffusion in porous media in the Knudsen regime, Phys. Rev. Lett. 87, 12, 125505.

5 Levitz P., Ehret G., Sinha S.K., Drake J.M. (1991) Porous vycor glass: the microstructure as probed by electron microscopy, direct energy transfer, small angle scattering, and molecular adsorption, J. Chem. Phys. 95, 8, 6151-6161.

6 Gregg S.J., Sing K.S.W. (1982) Adsorption, Surface Area and Porosimetry, Academic Press, New York.

7 Uhlir A. (1956) Electrolytic shaping of germanium and silicon, Bell Syst. Tech. J. 35, 333-347.

8 Barillaro G., Nannini A., Pieri F. (2003) APSFET: a new, porous silicon-based gas sensing device, Sens. Actuators B 93, 1, 263-270.

9 Meyers J.P., Maynard H.L. (2002) Design considerations for miniaturized PEM fuel cells, J. Power Sources 109, 1, 76-88. 
10 Grosman A., Ortega C., Wang Y.S., Gandais M. (1997) Morphology and structure of p-type porous silicon by transmission electron microscopy, in Structural and optical properties of Porous Silicon Nanostructures, Amato G., Delerue C., Von Bardeleben H.J. (eds), Gordon and Breach Science, London, pp. 317-331.

11 Grosman A., Ortega C. (2008) Capillary condensation in porous materials. Hysteresis and interaction mechanism without pore blocking/percolation process, Langmuir 24, 8, 3977-3986.

12 Coasne B., Grosman A., Ortega C., Simon M. (2002) Adsorption in noninterconnected pores open at one or at both ends: a reconsideration of the origin of the hysteresis phenomenon, Phys. Rev. Lett. 88, 25, 256102.

13 Dolino G., Bellet D., Faivre C. (1996) Adsorption strains in porous silicon, Phys. Rev. B 54, 24, 17919-17929.

14 Grosman A., Puibasset J., Rolley E. (2015) Adsorption-induced strain of a nanoscale silicon honeycomb, EPL 109, 5, 56002.

15 Lysenko V., Vitiello J., Remaki B., Barbier D. (2004) Gas permeability of porous silicon nanostructures, Phys. Rev. E 70, 1, 017301.

16 Naumov S., Khokhlov A., Valiullin R., Kärger J., Monson P.A. (2008) Understanding capillary condensation and hysteresis in porous silicon: Network effects within independent pores, Phys. Rev. E 78, 6, 060601.

17 Mason G. (1983) A model of adsorption - desorption hysteresis in which hysteresis is primarily developed by the interconnections in a network of pores, Proc. R. Soc. London A 390, 47-72.

18 Puibasset J. (2007) Adsorption/desorption hysteresis of simple fluids confined in realistic heterogeneous silica mesopores of micrometric length: a new analysis exploiting a multiscale Monte Carlo approach, J. Chem. Phys. 127, 15, 154701.
19 Grosman A., Ortega C. (2008) Influence of elastic deformation of porous materials in adsorption-desorption process: A thermodynamic approach, Phys. Rev. B 78, 085433.

20 Koponen A., Kataja M., Timonen J. (1996) Tortuous flow in porous media, Phys. Rev. E 54, 1, 406.

21 Stejskal E.O., Tanner J.E. (1965) Spin diffusion measurements: spin echoes in the presence of a time-dependent field gradient, J. Chem. Phys. 42, 288-292.

22 Callaghan P.T. (1991) Principles of Nuclear Magnetic Resonance Microscopy, Clarendon Press, Oxford.

23 Stallmach F., Kärger J., Krause C., Jeschke M., Oberhagemann U. (2000) Evidence of anisotropic self-diffusion of guest molecules in nanoporous materials of MCM-41 type, J. Am. Chem. Soc. 122, 38, 9237-9242.

24 Porion P., Faugère A.M., Delville A. (2008) ${ }^{1} \mathrm{H}$ and ${ }^{7} \mathrm{Li}$ NMR pulsed gradient spin echo measurements and multiscale modeling of the water and ionic mobility within aqueous dispersions of charged anisotropic nanoparticles, J. Phys. Chem. C 112, 31, 11893-11900.

25 Naumov S., Valiullin R., Kärger J., Pitchumani R., Coppens M.-O. (2008) Tracing pore connectivity and architecture in nanostructured silica SBA-15, Micropor. Mesopor. Mater. 110, $37-40$.
Manuscript submitted in July 2015 Manuscript accepted in November 2015

Published online in June 2016 